\title{
Difference in characteristics and outcomes between medullary breast carcinoma and invasive ductal carcinoma: a population based study from SEER 18 database
}

\author{
Xiao-Xiao Wang1, ${ }^{1,}$, Yi-Zhou Jiang ${ }^{2, *}$, Xi-Yu Liu ${ }^{2, *}$, Jun-Jing Li ${ }^{1}$, Chuan-Gui Song ${ }^{1}$, \\ Zhi-Ming Shao' \\ ${ }^{1}$ Department of Breast Surgery, Affiliated Union Hospital, Fujian Medical University, Fuzhou, China \\ ${ }^{2}$ Department of Breast Surgery, Key Laboratory of Breast Cancer, Fudan University Shanghai Cancer Center, Shanghai \\ Medical College, Fudan University, Shanghai, China \\ *These authors contributed equally to this work
}

Correspondence to: Chuan-Gui Song, e-mail: songchuangui@yahoo.com

Keywords: medullary breast carcinoma, invasive ductal carcinoma, breast cancer-specific survival, overall survival

Received: November 09, 2015

Accepted: February 18, 2016

Published: March 17, 2016

\section{ABSTRACT}

Medullary breast carcinoma (MBC) is a unique histological subtype of breast cancer. Our study was designed to identify difference in characteristics and outcomes between MBC and invasive ductal carcinoma (IDC), and further confirm the prognostic factors of MBC. Utilizing Surveillance, Epidemiology, and End Results (SEER), we identified 84,764 eligible patients, including 309 MBC and 84,455 IDC. Compared with the IDC group, the MBC group was associated with younger age at diagnosis, higher grade, more advanced stage, larger tumor size, and higher proportion of triplenegative breast cancer (TNBC). Kaplan-Meier analysis and univariate Cox proportional hazard regression model showed that patients with IDC had significantly better breast cancer-specific survival (BCSS) compared to MBC, but they had similar overall survival (OS). However, MBC histology was no longer a surrogate for worse BCSS or OS after 1:1 matching by age, American Joint Committee on Cancer (AJCC) stage, grade and breast subtype. In addition, it was exposed that not married status, high grade, large tumor size, positive nodal status, the subtype of TNBC and no receipt of radiation therapy were significantly associated with poor BCSS and OS. In conclusion, MBC demonstrated more aggressive behavior but similar outcomes compared to IDC, which may be determined by prognostic factors such as breast subtype. These results not only confer deeper insight into MBC but contribute to individualized and tailored therapy, and thereby may improve clinical management and outcomes.

\section{INTRODUCTION}

Medullary breast carcinoma (MBC) accounts for less than $5 \%$ of all invasive breast cancers. The clinicopathological characteristics and outcomes in MBC make it unique from infiltrating ductal carcinoma-not otherwise specified (IDC-NOS) [1].

$\mathrm{MBC}$ is characterized by a young age, a large tumor size, a high nuclear grade [2-4]. And some studies found that the MBCs seemed to exhibit a significantly higher proportion of triple-negative phenotype (absence of estrogen receptor (ER), progesterone receptor (PR), and human epidermal growth factor receptor-2 (HER-2)) based on a gene expression analysis or immunohistochemical staining
$[5,6]$. As is known to all, triple-negative breast cancer (TNBC) usually is relevant to a worse prognosis. However, though patients with $\mathrm{MBC}$ show aggressive histological features, there is no consensus regarding difference of clinicopathological characteristics and outcomes between MBC and IDC. Some studies have revealed that MBC histology is associated with a favorable prognosis $[2,5$, 7-9]. Other studies did not confirm this conclusion and even some indicated the prognosis of MBC did not differ from IDC [10-12].

Identification of prognostic factors is important for $\mathrm{MBC}$ patients so as to not only dictate diagnosis but also provide more appropriate treatment. Martinez et al. reported that in multivariate analysis, advanced age, black 
race, regional metastases, distant metastases, increased tumor size, ER positivity and increased lymph node metastasis (LNM) were associated with decreased overall survival in a cohort of MBC patients [13]. Park et al. identified there was no difference in disease-free survival (DFS) or overall survival (OS) among MBC patients according to tumor size, hormone receptor status, HER2 status, or adjuvant treatment but patients with lymph nodes metastasis presented a worse DFS and OS [12]. Based on small numbers of patients, different populations of studies and lacked adjustment of confounds, these prognostic factors specific to $\mathrm{MBC}$ are relatively unclear.

Given the controversial data on the prognosis of $\mathrm{MBC}$, this study was designed to identify difference in characteristics and outcomes between MBC and IDC, and further confirm the factors related to prognosis of $\mathrm{MBC}$ by utilizing Surveillance, Epidemiology, and End Results (SEER), a large population-based database.

\section{RESULTS}

\section{Demographics and clinical characteristics of study population}

A total of 84,764 patients met the eligibility criteria for our study, including 309 (0.36\%) MBC patients and $84,455(99.64 \%)$ IDC patients. Table 1 summarizes all of the demographic and clinical characteristics by histological subtype. There were significant differences in the characteristics including age of diagnosis, race, grade, American Joint Committee on Cancer (AJCC) stage, tumor size and breast subtype. MBC patients showed a younger age at diagnosis $(42.1 \%$ vs. $24.0 \%, P<0.001)$ and a poorer grade (grade III and IV, $93.5 \%$ vs. $36.2 \%, P<0.001$ ) than IDC patients. In addition, MBCs were much more likely to have a significantly higher proportion of black race $(23.3 \%$ vs. $10.4 \%, P<0.001)$ and the AJCC stage of II (52.8\% vs. $33.8 \%, P<0.001)$ than IDCs. Compared with IDC patients, MBC patients had larger tumor size (more tumors $>2 \mathrm{~cm}$ and $\leq 5 \mathrm{~cm}$ in size, $50.2 \%$ vs. $29.2 \%, P<0.001$ ). With regard to breast subtype, the MBC patients seemed more inclined to present TNBC compared with the IDC patients $(56.0 \%$ vs. $13.1 \%, P<0.001)$. The other tumor characteristics, including married status, laterality, lymph node (LN) status, types of surgery and radiation therapy were similarly distributed between the two histological types.

\section{Comparison of survival between MBCs and IDCs}

Kaplan-Meier analysis was used to evaluate breast cancer-specific survival (BCSS) and overall survival (OS) in these two histological types (Figure 1). As it illustrated, patients with IDC had better survival than MBC patients in BCSS $(P=0.013)$, but they had similar OS $(P=0.184)$.
In order to further investigate the prognostic factors, the results of the BCSS and OS analyses via univariate and multivariate Cox proportional hazard regression models were demonstrated in Supplementary Table S1 and Table 2, respectively. According to univariate analysis, not married status, grade III and IV, a tumor size of $>5 \mathrm{~cm}$, the increased number of lymph nodes, the subtype of TNBC and no receipt of radiation therapy were significantly associated with poor BCSS and OS, which were validated in the following multivariate analysis. However, after adjusting other prognostic factors, AJCC stage was no longer an independent prognostic factor in multivariate analysis. In the meantime, MBC patients showed significantly worse BCSS than IDC patients (univariate: hazard ratios, HRs $=2.483$, $95 \%$ confidence interval, CI: $1.180-5.228, P=0.017)$ and this result was no longer visible for OS.

\section{Survival estimates in matched group}

Considering the above confounding factors affecting breast cancer outcomes between MBC and IDC patients, we conducted a 1:1 (MBC/IDC) matched case-control analysis utilizing the propensity score matching method (Table 3 ). We obtained a group of 618 patients, including 309 patients for each histology type. For matched groups, no significant difference in characteristics was observed between MBC and IDC. Furthermore, we found that IDC histology no longer presented better prognosis for either BCSS or OS (Figure 2, $P=0.840$ and $P=0.513$ for BCSS and OS, respectively).

\section{Baseline characteristics and survival outcomes in triple-negative subgroup}

MBC patients predominantly had TNBC according to molecular subtype $(P<0.001)$. Therefore, we analyzed characteristics and survival outcomes of the patients limited to TNBC subgroup, which contained $173 \mathrm{MBC}$ patients and 11,056 IDC patients (Supplementary Table S2). And we came to some results in accordance with the entire population. For example, compared to TNBC-IDC patients, TNBC-MBC patients had younger age at diagnosis, larger proportion of black race and higher grade. Discriminately, nodal status and type of surgery become the significantly independent prognosis factors. However, AJCC stage and tumor size were no longer statistically different. In addition, the comparison of BCSS and OS by Kaplan-Meier curves presented similar survival for TNBC-MBC patients and TNBC-IDC patients (Figure 3, $P=0.504$ and $P=0.298$ for BCSS and OS, respectively). Furthermore, we matched 173 TNBC-MBC patients to 173 TNBC-IDC patients adjusted by age, AJCC stage and grade, utilizing the propensity score matching method. And we found BCSS and OS did not differ in the two groups (Figure 4, $P=0.812$ and $P=0.816$ for BCSS and OS, respectively). 
Table 1: Characteristics of patients with medullary breast carcinoma and invasive ductal carcinoma

\begin{tabular}{|c|c|c|c|c|c|c|c|c|}
\hline \multirow{2}{*}{\multicolumn{2}{|c|}{ Characteristics }} & \multicolumn{2}{|c|}{$\mathrm{MBC}(n=309)$} & \multicolumn{2}{|c|}{ IDC $(n=84455)$} & \multicolumn{2}{|c|}{ Total $(n=84764)$} & \multirow{2}{*}{$P^{\mathbf{c}}$} \\
\hline & & No. & $\%$ & No. & $\%$ & No. & $\%$ & \\
\hline \multicolumn{2}{|c|}{ Median follow-up (months) (IQR) } & \multicolumn{2}{|c|}{$17(9-27)$} & \multicolumn{2}{|c|}{$16(8-25)$} & \multicolumn{2}{|c|}{$16(8-25)$} & \\
\hline Age (years) & $18-49$ & 130 & 42.1 & 20297 & 24.0 & 20427 & 24.1 & $<0.001$ \\
\hline & $50-79$ & 179 & 57.9 & 64158 & 76.0 & 64337 & 75.9 & \\
\hline \multirow[t]{3}{*}{ Race } & White & 213 & 68.9 & 67456 & 79.9 & 67668 & 79.8 & $<0.001$ \\
\hline & Black & 72 & 23.3 & 8800 & 10.4 & 8872 & 10.5 & \\
\hline & Other $^{\mathrm{a}}$ & 24 & 7.8 & 8199 & 9.7 & 8223 & 9.7 & \\
\hline \multirow[t]{2}{*}{ Marital status } & Married & 185 & 59.9 & 52200 & 61.8 & 52385 & 61.8 & 0.484 \\
\hline & Not married ${ }^{b}$ & 124 & 40.1 & 32255 & 38.2 & 32379 & 38.2 & \\
\hline \multirow[t]{2}{*}{ Laterality } & Left & 150 & 48.5 & 42741 & 50.6 & 42891 & 50.6 & 0.469 \\
\hline & Right & 159 & 51.5 & 41714 & 49.4 & 41873 & 49.4 & \\
\hline \multirow[t]{3}{*}{ Grade } & I & 2 & 0.6 & 18452 & 21.8 & 18454 & 21.8 & $<0.001$ \\
\hline & II & 18 & 5.8 & 35405 & 41.9 & 35423 & 41.8 & \\
\hline & III and IV & 289 & 93.5 & 30598 & 36.2 & 30887 & 36.4 & \\
\hline \multirow[t]{3}{*}{ AJCC stage } & I & 126 & 40.8 & 48567 & 57.5 & 48693 & 57.4 & $<0.001$ \\
\hline & II & 163 & 52.8 & 28542 & 33.8 & 28705 & 33.9 & \\
\hline & III & 20 & 6.5 & 7346 & 8.7 & 7366 & 8.7 & \\
\hline \multirow[t]{3}{*}{ Tumor size $(\mathrm{cm})$} & $\leq 2$ & 141 & 45.6 & 56254 & 66.6 & 56395 & 66.5 & $<0.001$ \\
\hline & $>2$ and $\leq 5$ & 155 & 50.2 & 24645 & 29.2 & 24800 & 29.3 & \\
\hline & $>5$ & 13 & 4.2 & 3556 & 4.2 & 3569 & 4.2 & \\
\hline \multirow[t]{4}{*}{ Nodal status } & $\mathbf{0}$ & 240 & 77.7 & 60090 & 71.2 & 60330 & 71.2 & 0.087 \\
\hline & 1 to 3 & 54 & 17.5 & 18459 & 21.9 & 18513 & 21.3 & \\
\hline & 4 to 10 & 10 & 3.2 & 3998 & 4.7 & 4008 & 4.7 & \\
\hline & $>10$ & 5 & 1.6 & 1908 & 2.3 & 1913 & 2.3 & \\
\hline \multirow[t]{4}{*}{ Breast subtype } & HR+/Her2- & 99 & 32.0 & 60267 & 71.4 & 60366 & 71.2 & $<0.001$ \\
\hline & HR+/Her2+ & 10 & 3.2 & 9104 & 10.8 & 9114 & 10.8 & \\
\hline & HR-/Her2+ & 27 & 8.7 & 4028 & 4.8 & 4055 & 4.8 & \\
\hline & Triple negative & 173 & 56.0 & 11056 & 13.1 & 11229 & 13.2 & \\
\hline \multirow[t]{2}{*}{ Type of surgery } & BCS & 174 & 56.3 & 48311 & 57.2 & 48485 & 57.2 & 0.752 \\
\hline & Mastectomy & 135 & 43.7 & 36144 & 42.8 & 36279 & 42.8 & \\
\hline \multirow[t]{2}{*}{ Radiation } & No & 153 & 49.5 & 38055 & 45.1 & 38208 & 45.1 & 0.116 \\
\hline & Yes & 156 & 50.5 & 46400 & 54.9 & 46556 & 54.9 & \\
\hline
\end{tabular}

Abbreviation: MBC, medullary breast carcinoma; IDC, invasive ductal carcinoma; AJCC, American Joint Committee on Cancer; Her2, human epidermal growth factor receptor 2; HR, hormone receptor; BCS, breast conserving surgery; IQR, interquartile range.

${ }^{a}$ Other includes American Indian/Alaskan native, and Asian/Pacific Islander.

${ }^{b}$ Not married includes divorced, separated, single (never married), unmarried or domestic partner and widowed.

${ }^{c} P$ value was calculated among all groups by the Chi-square test, and a bold type indicates significance. 


\section{Stratification analysis with molecular subtype}

In order to further investigate the effects of molecular subtype on breast cancer outcomes between MBC and IDC patients, we performed multivariate analysis, stratifying according to molecular subtype. As shown in Table 4, when hazard ratios (HRs) of the two histological groups were conducted, no differences in BCSS and OS were observed, suggesting that molecular subtype may be a principal confounder for $\mathrm{MBC}$ prognosis.

\section{DISCUSSION}

In this large cohort of patients, we sought to reveal the difference in characteristics and outcomes between MBC and IDC, and summarize the prognostic factors of MBC by utilizing SEER population-based data. Our findings indicated that $\mathrm{MBC}$ had unique clinicalpathological characteristics and it was inclined to demonstrate more aggressive behavior over IDC. However, MBC histology presented similar prognosis in both BCSS and OS compared to IDC after adjusting and matching the confounding factors. Further stratification analysis indicated that breast cancer molecular subtype may be a principal confounder for MBC prognosis. In addition, it was exposed that married status, grade, tumor size, lymph node status, breast subtype and radiation therapy were significantly associated with BCSS and OS.

In our study, compared with the IDC group, the $\mathrm{MBC}$ group presented a younger age at diagnosis, higher grade, more advanced stage, larger tumor size, and higher proportion of TNBC. These observations were partially in concordance with previous studies. For example, Anderson et al. conducted an age-specific incidence rate curve and showed that MBC rates increased rapidly until age 50 and then failed to increase, while IDC rates increased rapidly until age 50 years and rose more slowly thereafter [14]. Park et al. demonstrated that women with MBC presented

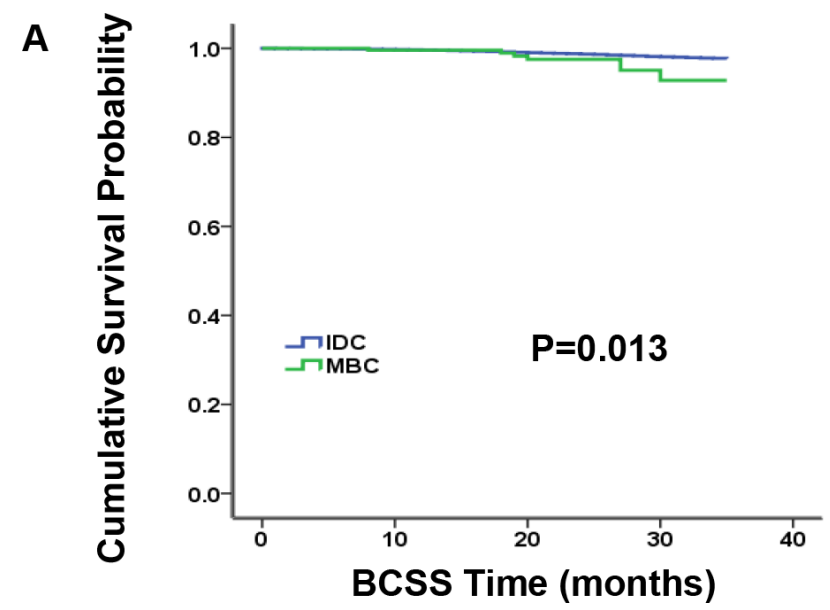

rare LN metastasis, ER and PR negativity, advanced tumor grade, and nuclear pleomorphism [12]. Vo et al. reported that a MBC group had larger tumors than an IDC group [10]. However, patients with MBC in China demonstrated less aggressive tumor features such as lower tumor stage, smaller tumor size and a lower proportion of nodal involvement than IDC [5].

Previous studies have revealed that $\mathrm{MBC}$ has a more favorable prognosis than IDC. For example, Huober et al. found that 14-year distant recurrence-free interval (DRFI), and overall survival (OS) percents for medullary tumors and invasive ductal tumors of the full cohort were $76 \%, 64 \%$ and $66 \%, 57 \%$, respectively [15]. However, some interesting findings observed in our study were that compared with IDC, MBC showed nearly the same outcomes as IDC in BCSS and OS after adjusting or matching the potential confounders. Collectively, these results implied that the MBC histological type was not an independent prognostic factor. Furthermore, among TNBC patients, MBC patients presented similar survival compared with IDC patients. Therefore, we speculated that breast cancer molecular subtype may be a principal confounder for $\mathrm{MBC}$ prognosis and it was further verified by stratification analysis with molecular subtype. In addition, we found that worse BCSS and OS for MBC compared to IDC were seen relevance to not married status, grade III and IV, a tumor size of $>5 \mathrm{~cm}$, the increased number of lymph nodes, the subtype of TNBC and no receipt of radiation therapy.

Inevitably, our study had several limitations. In terms of follow-up data, it is the well-known fact that information regarding HER-2 expression in SEER database was not available until 2010. We were therefore compelled to focus on the short-term survival status after initial diagnosis and identify any prognostic factors and an inadequate follow-up time may give rise to the skewed results. And beyond that, differences in treatments received could influence differences in survival, but information

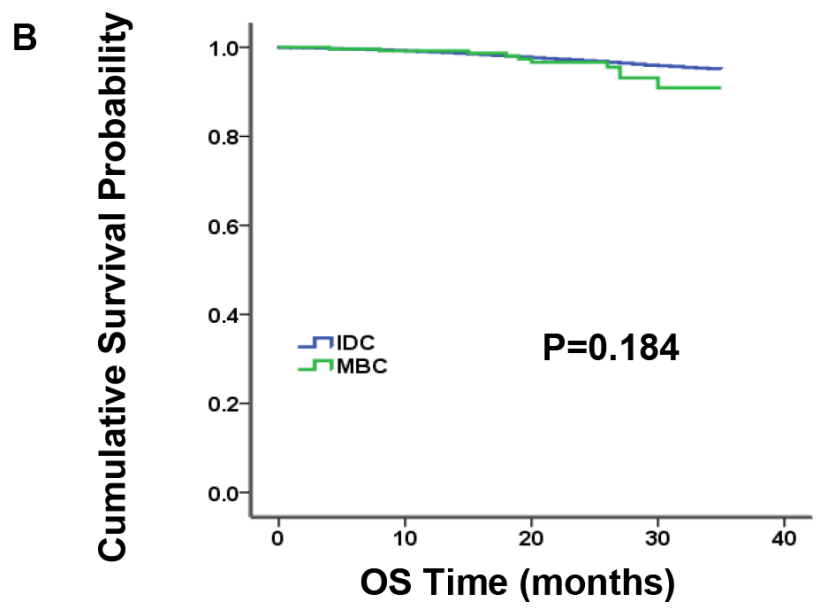

Figure 1: Kaplan-Meier plot and log-rank test compared breast cancer-specific survival (BCSS, $A$ ) and overall survival (OS, B) by histology for all patients, medullary breast carcinoma (MBC) vs. invasive ductal carcinoma (IDC). 
Table 2: Multivariate Cox proportional hazard model of breast cancer-specific survival (BCSS) and overall survival (OS)

\begin{tabular}{|c|c|c|c|c|c|}
\hline & & BCSS & & OS & \\
\hline & & HRs $(95 \% \mathrm{CI})$ & $P^{\mathbf{c}}$ & HRs $(05 \% \mathrm{CD})$ & $P_{c}$ \\
\hline Age (years) & $18-49$ & $0.825(0.701-0.971)$ & 0.020 & $0.545(0.480-0.618)$ & $<0.001$ \\
\hline & $50-79$ & Reference & & Reference & \\
\hline Race & White & Reference & & Reference & \\
\hline & Black & $1.144(0.946-1.383)$ & 0.165 & $1.078(0.944-1.232)$ & 0.267 \\
\hline & Other ${ }^{\mathrm{a}}$ & $0.610(0.442-0.842)$ & 0.003 & $0.668(0.544-0.819)$ & $<0.001$ \\
\hline Marital status & Married & Reference & & Reference & \\
\hline & Not married ${ }^{\mathrm{b}}$ & $1.290(1.111-1.497)$ & 0.001 & $1.562(1.417-1.722)$ & $<0.001$ \\
\hline Grade & I & $0.518(0.329-0.816)$ & 0.005 & $0.986(0.834-1.166)$ & 0.872 \\
\hline & II & Reference & & Reference & \\
\hline & III and IV & $2.719(1.779-2.670)$ & $<0.001$ & $1.360(1.941-2.403)$ & $<0.001$ \\
\hline Histology type & MBC & $1.304(0.490-2.183)$ & 0.931 & $1.518(1.206-1.533)$ & 0.188 \\
\hline & IDC & Reference & & Reference & \\
\hline AJCC stage & I & Reference & & Reference & \\
\hline & II & $1.385(0.976-1.965)$ & 0.068 & $0.909(0.730-1.131)$ & 0.390 \\
\hline & III & $1.530(0.890-2.630)$ & 0.124 & $0.923(0.618-1.377)$ & 0.694 \\
\hline Tumor size (cm) & $\leq 2$ & Reference & & Reference & \\
\hline & $>2$ and $\leq 5$ & $1.917(1.458-2.520)$ & $<0.001$ & $1.776(1.469-2.147)$ & $<0.001$ \\
\hline & $>5$ & $4.006(2.862-5.607)$ & $<0.001$ & $3.307(2.580-4.239)$ & $<0.001$ \\
\hline Nodal status & $\mathbf{0}$ & Reference & & Reference & \\
\hline & 1 to 3 & $1.860(1.499-2.308)$ & $<0.001$ & $1.453(1.260-1.676)$ & $<0.001$ \\
\hline & 4 to 10 & $3.011(1.939-4.674)$ & $<0.001$ & $2.405(1.693-3.415)$ & $<0.001$ \\
\hline & $>10$ & $5.248(3.440-8.005)$ & $<0.001$ & $3.673(2.607-5.175)$ & $<0.001$ \\
\hline Breast subtype & HR+/Her2- & Reference & & Reference & \\
\hline & HR+/Her2+ & $0.582(0.422-0.802)$ & 0.001 & $0.827(0.690-0.991)$ & 0.039 \\
\hline & HR-/Her2+ & $1.449(1.098-1.913)$ & 0.009 & $1.247(1.020-1.526)$ & 0.032 \\
\hline & Triple negative & $3.044(2.553-3.628)$ & $<0.001$ & $2.324(2.055-2.628)$ & $<0.001$ \\
\hline Type of surgery & BCS & Reference & & Reference & \\
\hline & Mastectomy & $1.037(0.875-1.229)$ & 0.672 & $0.589(0.769-0.959)$ & 0.007 \\
\hline Radiation & No & $1.796(1.533-2.105)$ & $<0.001$ & $2.396(2.147-2.675)$ & $<0.001$ \\
\hline & Yes & Reference & & Reference & \\
\hline
\end{tabular}

Abbreviation: AJCC, American Joint Committee on Cancer; MBC, medullary breast carcinoma; IDC, invasive ductal carcinoma; Her2, human epidermal growth factor receptor 2; HR, hormone receptor; BCS, breast conserving surgery; HRs, hazard ratios; CI, confidence interval; BCSS, breast cancer-specific survival; OS, overall survival.

${ }^{a}$ Other includes American Indian/Alaskan native, and Asian/Pacific Islander.

${ }^{b}$ Not married includes divorced, separated, single (never married), unmarried or domestic partner and widowed.

${ }^{c} P$ value was adjusted by multivariate Cox proportional hazard regression model including all factors, as categorized in Table 2 , and a bold type indicates significance. 
Table 3: Characteristics of patients with medullary breast carcinoma and invasive ductal carcinoma in 1:1 matched group

\begin{tabular}{|c|c|c|c|c|c|c|c|c|}
\hline \multirow{2}{*}{\multicolumn{2}{|c|}{ Characteristics }} & \multicolumn{2}{|c|}{$\operatorname{MBC}(n=309)$} & \multicolumn{2}{|c|}{ IDC $(n=309)$} & \multicolumn{2}{|c|}{ Total $(n=618)$} & \multirow{2}{*}{$P^{c}$} \\
\hline & & No. & $\%$ & No. & $\%$ & No. & $\%$ & \\
\hline \multicolumn{2}{|c|}{ Median follow-up (months) (IQR) } & \multicolumn{2}{|c|}{$17(9-27)$} & \multicolumn{2}{|c|}{$16(8-25)$} & \multicolumn{2}{|c|}{$17(8-26)$} & \\
\hline \multirow[t]{2}{*}{ Age (years) } & $18-49$ & 130 & 42.1 & 106 & 34.3 & 236 & 38.2 & 0.047 \\
\hline & $50-79$ & 179 & 57.9 & 203 & 65.7 & 382 & 61.8 & \\
\hline \multirow[t]{3}{*}{ Race } & White & 213 & 68.9 & 223 & 72.2 & 436 & 70.6 & 0.654 \\
\hline & Black & 72 & 23.3 & 63 & 20.4 & 135 & 21.8 & \\
\hline & Other $^{\mathrm{a}}$ & 24 & 7.8 & 23 & 7.4 & 47 & 7.6 & \\
\hline \multirow[t]{2}{*}{ Marital status } & Married & 185 & 59.9 & 194 & 62.8 & 379 & 61.3 & 0.457 \\
\hline & Not married $^{\mathrm{b}}$ & 124 & 40.1 & 115 & 37.2 & 239 & 38.2 & \\
\hline \multirow[t]{2}{*}{ Laterality } & Left & 150 & 48.5 & 157 & 50.8 & 307 & 49.7 & 0.573 \\
\hline & Right & 159 & 51.5 & 152 & 49.2 & 311 & 50.3 & \\
\hline \multirow[t]{3}{*}{ Grade } & I & 2 & 0.6 & 2 & 0.6 & 4 & 0.6 & 1.000 \\
\hline & II & 18 & 5.8 & 18 & 5.8 & 36 & 5.8 & \\
\hline & III and IV & 289 & 93.5 & 289 & 93.5 & 578 & 93.5 & \\
\hline \multirow[t]{3}{*}{ AJCC stage } & I & 126 & 40.8 & 126 & 40.8 & 252 & 40.8 & 1.000 \\
\hline & II & 163 & 52.8 & 163 & 52.8 & 362 & 52.8 & \\
\hline & III & 20 & 6.5 & 20 & 6.5 & 40 & 6.5 & \\
\hline \multirow[t]{3}{*}{ Tumor size $(\mathrm{cm})$} & $\leq 2$ & 141 & 45.6 & 148 & 47.9 & 289 & 46.8 & 0.666 \\
\hline & $>2$ and $\leq 5$ & 155 & 50.2 & 145 & 46.9 & 300 & 48.5 & \\
\hline & $>5$ & 13 & 4.2 & 16 & 5.2 & 29 & 4.7 & \\
\hline \multirow[t]{4}{*}{ Nodal status } & $\mathbf{0}$ & 240 & 77.7 & 222 & 71.8 & 462 & 74.8 & 0.216 \\
\hline & 1 to 3 & 54 & 17.5 & 75 & 24.3 & 129 & 20.9 & \\
\hline & 4 to 10 & 10 & 3.2 & 8 & 2.6 & 18 & 2.9 & \\
\hline & $>10$ & 5 & 1.6 & 4 & 1.3 & 9 & 1.5 & \\
\hline \multirow[t]{4}{*}{ Breast subtype } & HR+/Her2- & 99 & 32.0 & 99 & 32.0 & 198 & 32.0 & 1.000 \\
\hline & HR+/Her2+ & 10 & 3.2 & 10 & 3.2 & 20 & 3.2 & \\
\hline & HR-/Her2+ & 27 & 8.7 & 27 & 8.7 & 54 & 8.7 & \\
\hline & Triple negative & 173 & 56.0 & 173 & 56.0 & 346 & 56.0 & \\
\hline \multirow[t]{2}{*}{ Type of surgery } & BCS & 174 & 56.3 & 158 & 51.1 & 332 & 53.7 & 0.197 \\
\hline & Mastectomy & 135 & 43.7 & 151 & 48.9 & 286 & 46.3 & \\
\hline \multirow[t]{2}{*}{ Radiation } & No & 153 & 49.5 & 165 & 53.4 & 318 & 51.5 & 0.334 \\
\hline & Yes & 156 & 50.5 & 144 & 46.6 & 300 & 48.5 & \\
\hline
\end{tabular}

Abbreviation: MBC, medullary breast carcinoma; IDC, invasive ductal carcinoma; AJCC, American Joint Committee on Cancer; Her2, human epidermal growth factor receptor 2; HR, hormone receptor; BCS, breast conserving surgery; IQR, interquartile range.

${ }^{a}$ Other includes American Indian/Alaskan native, and Asian/Pacific Islander.

${ }^{b}$ Not married includes divorced, separated, single (never married), unmarried or domestic partner and widowed.

${ }^{c} P$ value was calculated among all groups by the Chi-square test after matching on age, grade, AJCC stage, breast subtype, and a bold type indicates significance. 

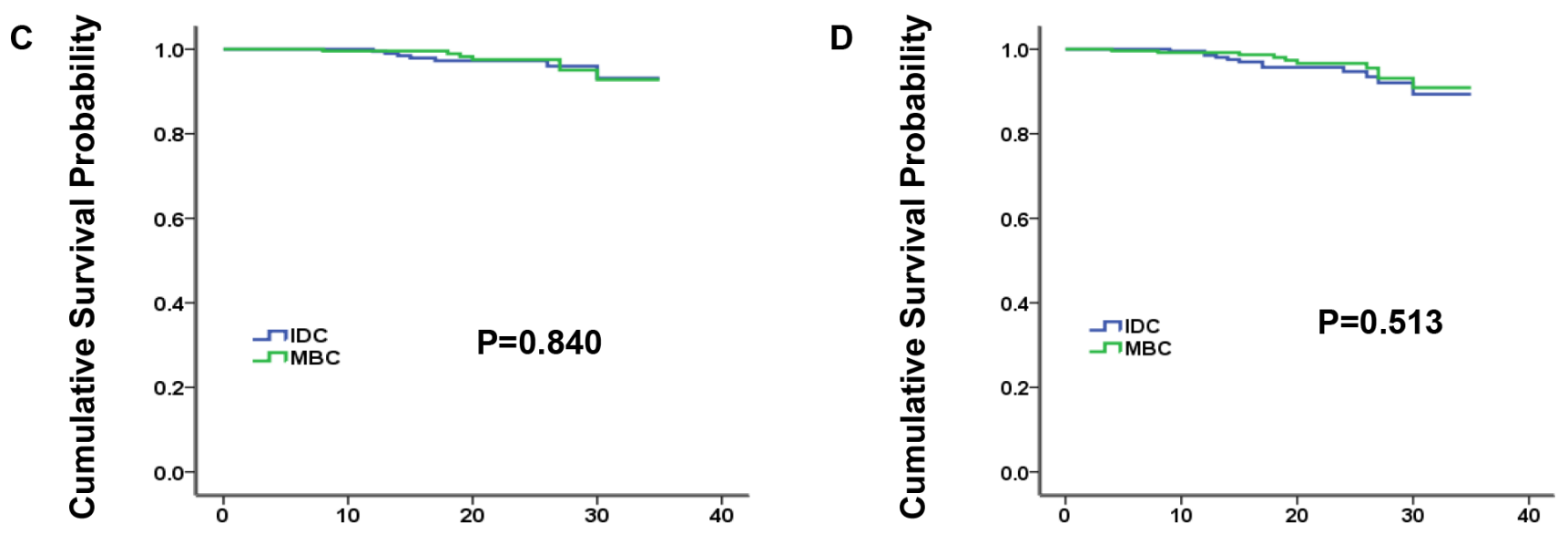

Figure 2: Kaplan-Meier plot and log-rank test compared breast cancer-specific survival (BCSS, C) and overall survival (OS, D) by histology for 1:1 matched group, medullary breast carcinoma (MBC) vs. invasive ductal carcinoma (IDC).
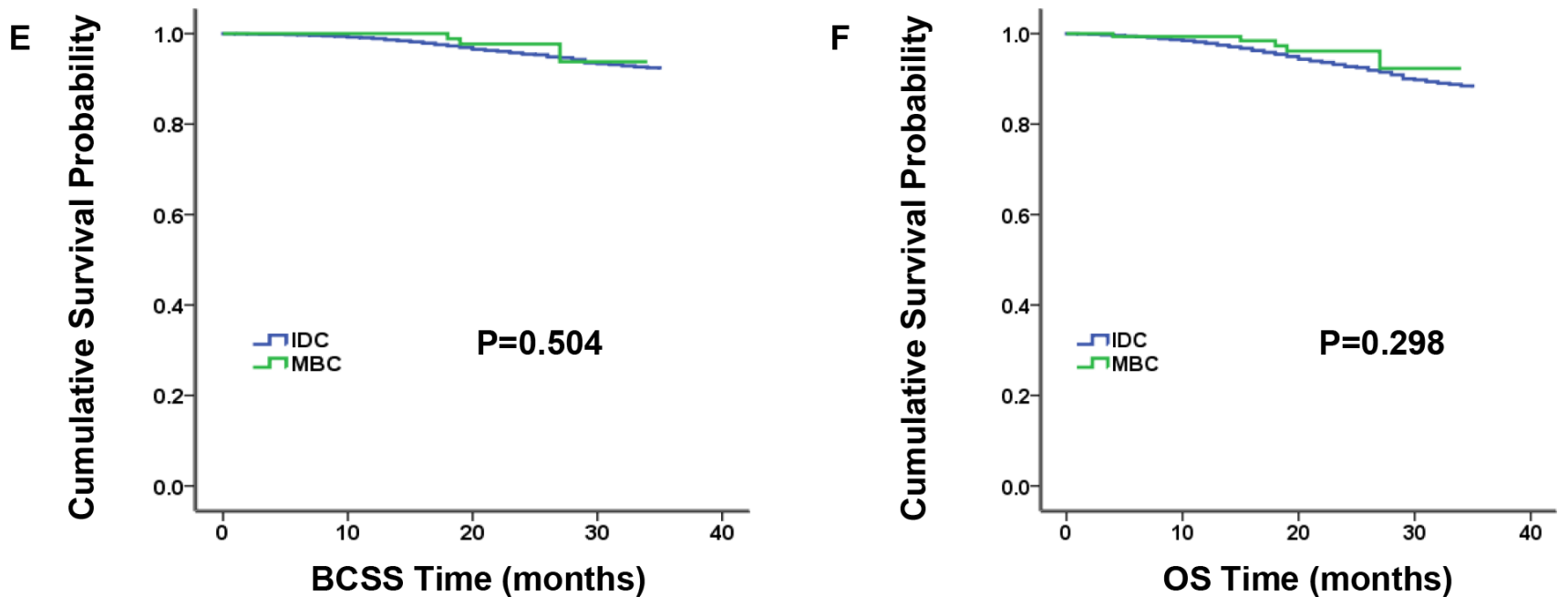

Figure 3: Kaplan-Meier plot and log-rank test compared breast cancer-specific survival (BCSS, E) and overall survival (OS, F) by histology for triple-negative breast cancer (TNBC) patients, medullary breast carcinoma (MBC) vs. invasive ductal carcinoma (IDC).
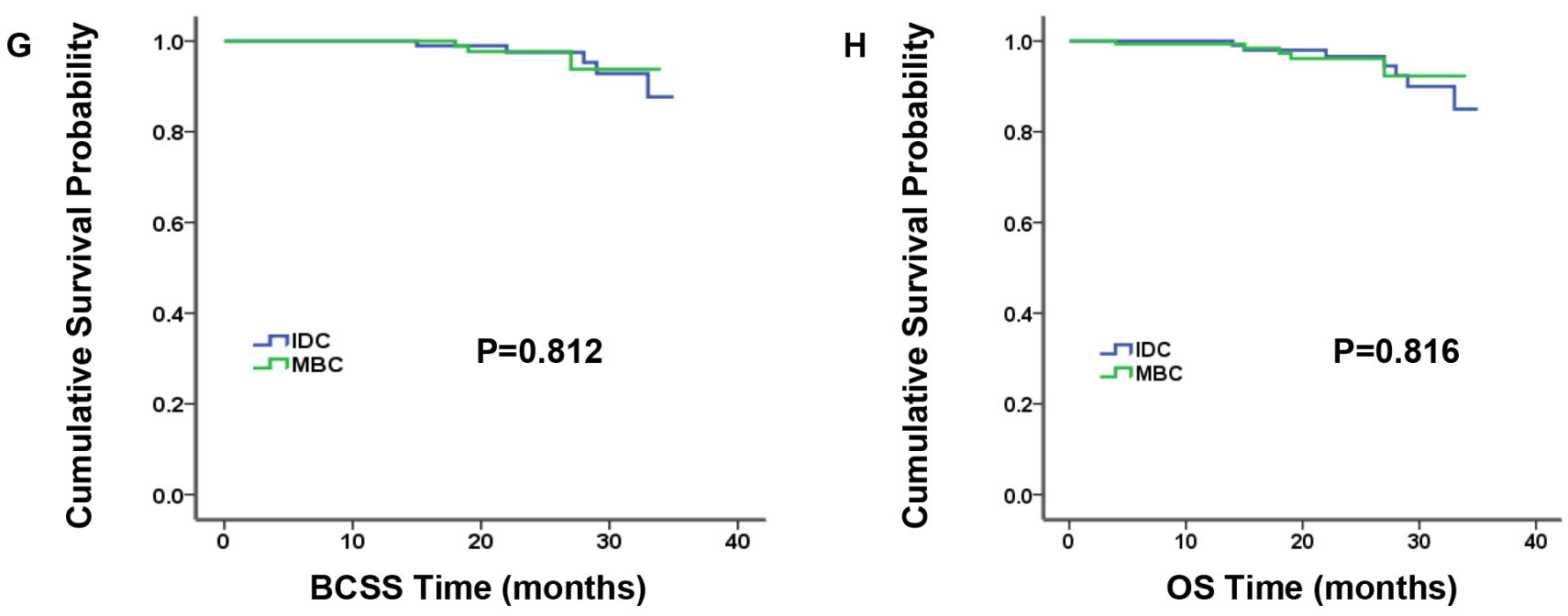

Figure 4: Kaplan-Meier plot and log-rank test compared breast cancer-specific survival (BCSS, G) and overall survival (OS, H) by histology for 1:1 matched triple-negative breast cancer (TNBC) patients, medullary breast carcinoma (MBC) vs. invasive ductal carcinoma (IDC). 
Table 4: Comparison of breast cancer-specific survival (BCSS) and overall survival (OS) between medullary breast carcinoma and invasive ductal carcinoma after subgroup analyses by multivariate Cox proportional hazard model

\begin{tabular}{|c|c|c|c|c|c|c|}
\hline \multirow{2}{*}{ Subtype } & \multicolumn{3}{|c|}{ BCSS } & \multicolumn{3}{|c|}{ OS } \\
\hline & Events No. & HRs (95\% CI) & $P^{\mathbf{a}}$ & Events No. & HRs (95\% CI) & $P^{\mathbf{a}}$ \\
\hline HR+/Her2- & & & 0.492 & & & 0.686 \\
\hline $\operatorname{MBC}(n=99)$ & 2 & $1.632(0.404-6.595)$ & & 3 & $1.265(0.406-3.945)$ & \\
\hline IDC $(n=60267)$ & 282 & Reference & & 886 & Reference & \\
\hline HR $+/$ Her2+ & & & 0.997 & & & 0.962 \\
\hline $\operatorname{MBC}(n=10)$ & 0 & - & & 0 & - & \\
\hline IDC $(n=9104)$ & 44 & Reference & & 142 & Reference & \\
\hline HR-/Her2+ & & & 0.581 & & & 0.971 \\
\hline $\operatorname{MBC}(n=27)$ & 1 & $1.755(0.239-12.892)$ & & 1 & $1.037(0.144-7.494)$ & \\
\hline IDC $(n=4028)$ & 64 & Reference & & 115 & Reference & \\
\hline Triple negative & & & 0.742 & & & 0.535 \\
\hline $\operatorname{MBC}(n=173)$ & 4 & $0.847(0.315-2.275)$ & & 6 & $0.775(0.346-1.735)$ & \\
\hline IDC $(n=11094)$ & 329 & Reference & & 541 & Reference & \\
\hline
\end{tabular}

Abbreviation: MBC, medullary breast carcinoma; IDC, invasive ductal carcinoma; HR, hormone receptor; Her2, human epidermal growth factor receptor 2; HRs, hazard ratios; CI, confidence interval; BCSS, breast cancer-specific survival; OS, overall survival.

${ }^{a} P$ value was adjusted by multivariate Cox proportional hazard regression model including age, race, marital statuses, grade, tumor size, lymph node status, type of surgery, radiation and a bold type indicates significance.

regarding adjuvant or neoadjuvant chemotherapy is absent from the SEER database.

In conclusion, our investigations revealed that MBCs have unique clinicopathological characteristics, including a younger age at diagnosis, higher grade, more advanced stage, larger tumor size, and higher proportion of TNBC. However, MBC presented similar prognosis in both BCSS and OS compared with IDC. These results not only confer deeper insight into MBC but contribute to clinical practice that doctors ought to provide patients with MBC the same intensive treatment as those with IDC and, thereby may improve clinical management and outcomes.

\section{MATERIALS AND METHODS}

\section{Patients}

We used SEER * Stat version 8.2.1 to generate a case listing. We identified 84,764 eligible patients according to the following inclusion criteria: female, year of diagnosis from 2010 to 2012, age of diagnosis between 18 and 79 years, breast cancer as the first and only malignant cancer diagnosis, pathologically confirmed medullary breast carcinoma, not otherwise specified (MBC-NOS, ICD-O-3 $8510 / 3$ ) or invasive ductal carcinoma, not otherwise specified (IDC-NOS, ICD-O-3 8500/3), unilateral cancer, breast subtype, histological grades I to IV, AJCC TNM stages I-III, surgical treatment with either mastectomy or breastconserving surgery, known ER, PR and HER2 statuses. We excluded patients who were diagnosed with breast cancer at death or by autopsy only and those with other first primary cancers, in situ disease, and no record of surgery type or radiation therapy. Patients diagnosed with breast cancer before 2010 were not included because the SEER database did not record data on HER2 status until 2010. Additionally, patients diagnosed with breast cancer after 2012 were not included because the database was only updated through December 31, 2012, and we wanted to ensure an adequate follow-up duration. We calculated follow-up times from January 1, 2010 to December 31, 2012.

\section{Statistical analysis}

The demographics and clinical characteristics of incorporated cases were compared between MBC and IDC groups with the Chi square test. Kaplan-Meier method 
was performed to generate the survival curves, and the log-rank test was performed to compare the unadjusted BCSS and OS rates of patients with different histological subtype. BCSS was measured from the date of diagnosis to the date of breast cancer death. OS was defined as the time from the date of diagnosis to the date of death due to all causes (including breast cancer) or the last followup. Adjusted HRs with 95\% CIs were calculated using Cox proportional hazard regression models in order to estimate the prognostic factors. These above statistical analyses were performed utilizing SPSS version 20.0 software package (IBM SPSS Statistics, Chicago, IL, US). In addition, we matched each MBC patient to 1 IDC patient on the following predetermined factors: age, AJCC stage, grade, breast subtype, utilizing psmatch2 in Stata, version 12.0 (StataCorp, College Station, TX) designed for the propensity score matching methods and tested the matching quality for the balance after the match. Two-sided $P$-value $<0.05$ was considered statistically significant.

\section{ACKNOWLEDGMENTS AND FUNDING}

This work was supported by grants from the Training Plan of Middle-aged and Young Talents of Fujian Province Health and Family Planning Commission (2014-ZQN-ZD-10); the Provincial Natural Science Foundation of Fujian, China (2012J01357); the Research Project of Fudan University Shanghai Cancer Center (YJ201401), the National Natural Science Foundation of China (81572583, 81502278, 81372848, 81370075); the Municipal Project for Developing Emerging and Frontier Technology in Shanghai Hospitals (SHDC12010116); the Cooperation Project of Conquering Major Diseases in Shanghai Municipality Health System (2013ZYJB0302); the Innovation Team of Ministry of Education (IRT1223); and the Shanghai Key Laboratory of Breast Cancer (12DZ2260100). The funders had no role in the study design, data collection and analysis, decision to publish, or preparation of the manuscript.

\section{CONFLICTS OF INTEREST}

The authors have declared that no competing interests exist.

\section{REFERENCES}

1. Pedersen L, Zedeler K, Holck S, Schiodt T, Mouridsen HT. Medullary carcinoma of the breast. Prevalence and prognostic importance of classical risk factors in breast cancer. Eur $\mathrm{J}$ Cancer. 1995; 31A:2289-2295.

2. Vu-Nishino H, Tavassoli FA, Ahrens WA, Haffty BG. Clinicopathologic features and long-term outcome of patients with medullary breast carcinoma managed with breast-conserving therapy (BCT). Int J Radiat Oncol Biol Phys. 2005; 62:1040-1047.
3. Li CI. Risk of mortality by histologic type of breast cancer in the United States. Horm Cancer. 2010; 1:156-165.

4. Li CI, Uribe DJM Daling JR. Clinical characteristics of different histologic types of breast cancer. Brit J Cancer. 2005; 93:1046-1052.

5. Cao AY, He M, Huang L, Shao ZM, Di GH. Clinicopathologic characteristics at diagnosis and the survival of patients with medullary breast carcinoma in China_ a comparison with infiltrating ductal carcinoma-not otherwise specified. World J Surg Oncol. 2013; 11:91.

6. Bertucci F, Finetti P, Cervera N, Charafe-Jauffret E, Mamessier E, Adelaide J, Debono S, Houvenaeghel G, Maraninchi D, Viens P, Charpin C, Jacquemier J, Birnbaum D. Gene expression profiling shows medullary breast cancer is a subgroup of basal breast cancers. Cancer Res. 2006; 66:4636-4644.

7. Ridolfi RL, Rosen PP, Port A, Kinne D, Mike V. Medullary carcinoma of the breast: a clinicopathologic study with 10 year follow-up. Cancer. 1977; 40:1365-1385.

8. Jensen ML, Kiaer H, Andersen J, Jensen V, Melsen F. Prognostic comparison of three classifications for medullary carcinomas of the breast. Histopathology. 1997; 30:523-532.

9. Rakha EA, Putti TC, Abd El-Rehim DM, Paish C, Green AR, Powe DG, Lee AH, Robertson JF, Ellis IO. Morphological and immunophenotypic analysis of breast carcinomas with basal and myoepithelial differentiation. J Pathol. 2006; 208:495-506.

10. Vo T, Xing Y, Meric-Bernstam F, Mirza N, Vlastos G, Symmans WF, Perkins GH, Buchholz TA, Babiera GV, Kuerer HM, Bedrosian I, Akins JS, Hunt KK. Long-term outcomes in patients with mucinous, medullary, tubular, and invasive ductal carcinomas after lumpectomy. Am J Surg. 2007; 194:527-531.

11. Fisher ER, Kenny JP, Sass R, Dimitrov NV, Siderits RH, Fisher B. Medullary cancer of the breast revisited. Breast Cancer Res Treat. 1990; 16:215-229.

12. Park I, Kim J, Kim M, Bae SY, Lee SK, Kil WH, Lee JE, Nam SJ. Comparison of the characteristics of medullary breast carcinoma and invasive ductal carcinoma. J Breast Cancer. 2013; 16:417-425.

13. Martinez SR, Beal SH, Canter RJ, Chen SL, Khatri VP, Bold RJ. Medullary carcinoma of the breast: a populationbased perspective. Med Oncol. 2011; 28:738-744.

14. Anderson WF, Chu KC, Chang S, Sherman ME. Comparison of age-specific incidence rate patterns for different histopathologic types of breast carcinoma. Cancer Epidemiol Biomarkers Prev. 2004; 13:1128-1135.

15. Huober J, Gelber S, Goldhirsch A, Coates AS, Viale G, Ohlschlegel C, Price KN, Gelber RD, Regan MM, Thurlimann B. Prognosis of medullary breast cancer: analysis of 13 International Breast Cancer Study Group (IBCSG) trials. Ann Oncol. 2012; 23:2843-2851. 